\title{
Molecular Dynamics of Proteins Investigated by NMR Relaxation Methods
}

\author{
D. WierzUCHOWSKA ${ }^{a, *}$ AND B. Blicharska ${ }^{b}$ \\ ${ }^{a}$ Institute of Physics, Pedagogical University, Podchorążych 2, 30-084 Kraków, Poland \\ ${ }^{b}$ Institute of Physics, Jagiellonian University, W.S. Reymonta 4, 30-059 Kraków, Poland
}

\begin{abstract}
The nuclear magnetic resonance relaxation times of solvent water nuclei are known to decrease upon addition of diamagnetic solute protein. For this reason NMR relaxation methods are able to provide information on molecular dynamics changes of water protons and their interaction with macromolecules' surfaces. We present results of measurements of relaxation rates $R_{1}=1 / T_{1}, R_{2}=1 / T_{2}$ and $R_{1 \rho}=1 / T_{1 \rho}$ in the rotating frame for three proteins: chicken egg white lysozyme, egg white albumin, and bovine serum albumin, obtained at proton resonant frequency of $60 \mathrm{MHz}$. Besides the relaxation rates dependences on concentration in the $4-23 \%(\mathrm{~g} / 100 \mathrm{~g}$ solution) range, the analysis of the Carr-Purcell-Meiboom-Gill CPMG multi-echo $T_{2}$ experiments with variable pulse rate $\tau$ was performed. The dependences of relaxation rates on protein concentration are linear at low concentration. When protein concentration increases the slope of the straight line rapidly changes at so-called "critical" concentration which depends on MW of the diluted protein. Investigated dispersion of $T_{2}$, obtained using the CPMG method with a variable pulse rate, for concentrations higher and lower than the "critical" one, exhibits unequal behavior. At high concentration one-exponential curves and at low concentration two-exponential curves correspond closely with experimental data. The obtained parameters of exponents allow an estimation of the ratio of the amount of water with the determined motion freedom, that is free and bounded water, in solution. We showed that the CPMG dispersion method applied to aqueous protein solutions may widen the current understanding of the nature of molecular dynamics of hydrated water protons in non-perturbed environment.
\end{abstract}

DOI: 10.12693 /APhysPolA.125.907

PACS: $76.60 .-\mathrm{k}, 76.90+\mathrm{d}$

\section{Introduction}

NMR relaxation methods exploit the common $T_{1}$ and $T_{2}$ relaxation times measurements and their dependences on concentration, as well as new dispersion methods which have been lately introduced. There are two dispersion methods which are currently often used: measurements of relaxation time in rotating frame $T_{1 \rho}$ dependence on magnetic field $B_{1}[1]$ and the Carr-PurcellMeiboom-Gill (CPMG) $T_{2}$ dependence on pulse frequency (or time delay $\tau$ between $\pi$ pulses in CPMG sequence) [2]. These methods are useful tools for studying conformation changes and molecular dynamics over a wide time scale extending from picoseconds to seconds. Molecular dynamics affects the rate and pathway of protein folding as well as misfolding and aggregation can inhibit some proteins from performing multiple district function and are important for the evolution of novel function. Admittedly NMR methods do not always offer certain and direct insight into protein structure, but they offer a non-perturbing look into the nature of solution, a wide range of application and a substantial theoretical basis [3].

In what follows we present first of all the CPMG relaxation dispersion for studies of millisecond time dynamics in aqueous solutions of egg white lysozyme, egg white albumin (EWA), and bovine serum albumin

*corresponding author; e-mail: dw7@onet.eu
(BSA). Investigated substances are a few of many proteins which are important in several biochemical and biophysical processes and are well examined using various physico-chemical methods, but many questions concerning molecular dynamics remain unsolved $[4,5]$.

\section{Materials and methods}

Dry, lyophilized powder of egg white albumin (MW 44287 Da), was purchased from "Polskie Odczynniki Chemiczne" Company, egg white lysozyme (MW 14 600Da), and bovine serum albumin (MW 66382 Da) were purchased from Sigma Aldrich Company. Protein solutions at concentrations ranging from $4 \%$ to $23 \%(\mathrm{~g} /(100 \mathrm{~g}$ solution $))$ were prepared by dissolving protein powder in double distilled and de-ionized water. Temperature of the samples was stabilized to an accuracy $\pm 1{ }^{\circ} \mathrm{C}$. Measurements were performed at room temperature $+23^{\circ} \mathrm{C}$.

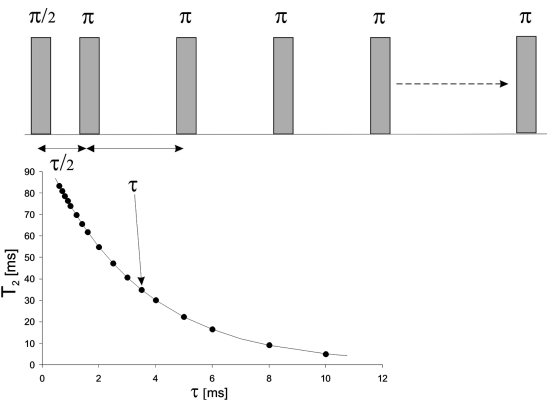

Fig. 1. CPMG pulse sequence and obtained dispersion curve $T_{2}(\tau)$. 
All samples were measured with Minispec Bruker spectrometer working at a resonant proton frequency of $60 \mathrm{MHz}$. $T_{1}$ was measured using the inversion recovery (IR) sequence. The standard spin-locking pulse sequence ( $\pi / 2-\tau$-spin-lock-FID) was used to measure $T_{1 \rho}$ [1].

Typical CPMG spin-echo pulse phase cycling with various pulse rate $\tau$ was used to measure $T_{2}$ dependence on $\tau$ (Fig. 1 ) where $\pi / 2$ pulses were $2.16 \pm 0.02 \mu$ s and $\pi$ pulses were $4.28 \pm 0.02 \mu$ s long. Obtained signals were accumulated 4-40 times depending on sample $\mathrm{S} / \mathrm{N}$ ratio. Repetition time was always longer than $5 T_{1}$.

Our CPMG dispersion measurements data are presented as $T_{2}(\tau)$ function.

\section{Results and discussion}

The results of measurements are presented in Table.

Values of $T_{1}$ and $T_{2}$ (at $\tau=1 \mathrm{~ms}$ ) for lysozyme, EWA, and BSA solutions at $+23{ }^{\circ} \mathrm{C}$.

TABLE

\begin{tabular}{|c|c|c|c|c|c|}
\hline \multirow[t]{2}{*}{ Sample } & \multirow{2}{*}{$\begin{array}{c}\text { Concentration } \% \\
{[\mathrm{~g} / 100 \mathrm{~g}]}\end{array}$} & \multirow[t]{2}{*}{$T_{1}[\mathrm{~ms}]$} & \multirow{2}{*}{$\begin{array}{c}T_{2}[\mathrm{~ms}] \\
\text { at } \tau=1 \mathrm{~ms}\end{array}$} & \multicolumn{2}{|c|}{$\begin{array}{c}\text { Parameters of fitted } \\
\text { exponential } T(\tau) \text { curves }\end{array}$} \\
\hline & & & & $a$ & $b$ \\
\hline Lysozyme & 21.45 & $1190 \pm 30$ & $449 \pm 3$ & $1.15 \pm 0.33$ & \\
\hline \multirow{10}{*}{$\begin{array}{c}\text { critical } \\
\text { concentration } \\
17 \pm 1 \%\end{array}$} & 20.55 & $1260 \pm 30$ & $493 \pm 2$ & $1.12 \pm 0.1$ & \\
\hline & 18.11 & $1400 \pm 30$ & $595 \pm 1$ & $1.18 \pm 0.1$ & \\
\hline & 15.54 & $1760 \pm 35$ & $932 \pm 2$ & $1.09 \pm 0.1$ & \\
\hline & 12.2 & $1821 \pm 20$ & $884 \pm 1$ & $0.88 \pm 0.11$ & $12.56 \pm 11$ \\
\hline & 9.38 & $2045 \pm 20$ & $1096 \pm 1$ & $0.90 \pm 0.1$ & $2.12 \pm 1.11$ \\
\hline & 8.05 & $2080 \pm 15$ & $1195 \pm 1$ & $0.98 \pm 0.22$ & $18.30 \pm 70$ \\
\hline & 7.71 & $2203 \pm 8$ & $1293 \pm 1$ & $0.73 \pm 0.37$ & $3.09 \pm 0.1$ \\
\hline & 6.94 & $2160 \pm 10$ & $1300 \pm 1$ & $0.89 \pm 0.3$ & $1.82 \pm 0.8$ \\
\hline & 6.16 & $2267 \pm 9$ & $1425 \pm 1$ & $0.88 \pm 0.40$ & $16 \pm 70$ \\
\hline & 5.40 & $2257 \pm 9$ & $1401 \pm 1$ & $0.98 \pm 0.25$ & $12.77 \pm 4$ \\
\hline EWA & 22.5 & $21038 \pm 8$ & $196 \pm 05$ & $\bar{~} 0.81 \pm 0.15$ & \\
\hline \multirow{13}{*}{$\begin{array}{c}\text { critical } \\
\text { concentration } \\
12.8 \pm 0.1 \%\end{array}$} & 20.26 & $1127 \pm 5$ & $246.9 \pm 0.2$ & $1.12 \pm 0.7$ & \\
\hline & 19.06 & $1194 \pm 9$ & $273.4 \pm 0.1$ & $0.98 \pm 0.2$ & \\
\hline & 18.77 & $1220 \pm 10$ & $283.5 \pm 0.4$ & $0.75 \pm 0.2$ & \\
\hline & 17.71 & $1244 \pm 5$ & $296.7 \pm 0.1$ & $1.07 \pm 0.07$ & \\
\hline & 14.25 & $1435 \pm 10$ & $382.8 \pm 0.1$ & $0.55 \pm 0.2$ & $1.77 \pm 0.32$ \\
\hline & 12.71 & $1645 \pm 67$ & $459.7 \pm 0.2$ & $0.66 \pm 01$ & $3.27 \pm 0.55$ \\
\hline & 9.68 & $1714 \pm 8$ & $550.7 \pm 0,2$ & $0.92 \pm 0.24$ & $2.47 \pm 5$ \\
\hline & 5.58 & $2057 \pm 4$ & $857 \pm 0.3$ & $0.97 \pm 0.2$ & $7.41 \pm 12$ \\
\hline & 5.03 & $2147 \pm 8$ & $958.3 \pm 0.1$ & $0.22 \pm 1$ & $1.91 \pm 12$ \\
\hline & 4.68 & $2258 \pm 4$ & $979.9 \pm 0,1$ & $0.69 \pm 0.17$ & $2.21 \pm 1.71$ \\
\hline & 4.26 & $2323 \pm 3$ & $1065.7 \pm 0.1$ & $0.76 \pm 0.19$ & $4.19 \pm 1.4$ \\
\hline & 3.71 & $2396 \pm 5$ & $1213.7 \pm 0.4$ & $0.89 \pm 0.11$ & $7.62 \pm 4.52$ \\
\hline & 2.2 & $2648 \pm 10$ & $1415 \pm 0.2$ & $0.33 \pm 0.24$ & $2.01 \pm 12$ \\
\hline$\overline{\mathrm{BSA}}$ & 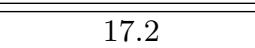 & 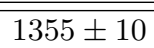 & 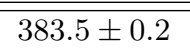 & 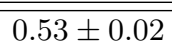 & \\
\hline \multirow{9}{*}{$\begin{array}{c}\text { critical } \\
\text { concentration } \\
7.2 \pm 0.5 \%\end{array}$} & 16.15 & $1430 \pm 30$ & $358 \pm 0.2$ & $0.90 \pm 0.03$ & \\
\hline & 13.59 & $1620 \pm 10$ & $460 \pm 0.4$ & $0.65 \pm 0.01$ & \\
\hline & 10.9 & $1792 \pm 20$ & $533 \pm 2$ & $0.62 \pm 0.03$ & \\
\hline & 8.23 & $2021 \pm 20$ & $681 \pm 0.1$ & $0.48 \pm 0.03$ & $2.73 \pm 0.25$ \\
\hline & 7.84 & $2100 \pm 6$ & $713 \pm 0.6$ & $0.69 \pm 0.07$ & $13.69 \pm 17$ \\
\hline & 7.31 & $2178 \pm 20$ & $748 \pm 1$ & $0.43 \pm 0.15$ & $2.09 \pm 0.56$ \\
\hline & 6.23 & $2235 \pm 20$ & $854 \pm 0.5$ & $0.62 \pm 0.13$ & $2.79 \pm 0.5$ \\
\hline & 5.95 & $2244 \pm 3$ & $871 \pm 1$ & $0.66 \pm 0.09$ & $7.72 \pm 7$ \\
\hline & 4.33 & $2393 \pm 10$ & $1111.6 \pm 0.5$ & $0.62 \pm 0.07$ & $5.13 \pm 3.5$ \\
\hline
\end{tabular}

The relaxation rates $R_{1}, R_{2}$, and $R_{1 \rho}$ dependences on concentration for EWA solutions are plotted in Fig. $2 \mathrm{a}-\mathrm{c}$.
An example of $T_{1}$ dependence on concentration for BSA is presented in Fig. 3. "Critical" concentrations for 


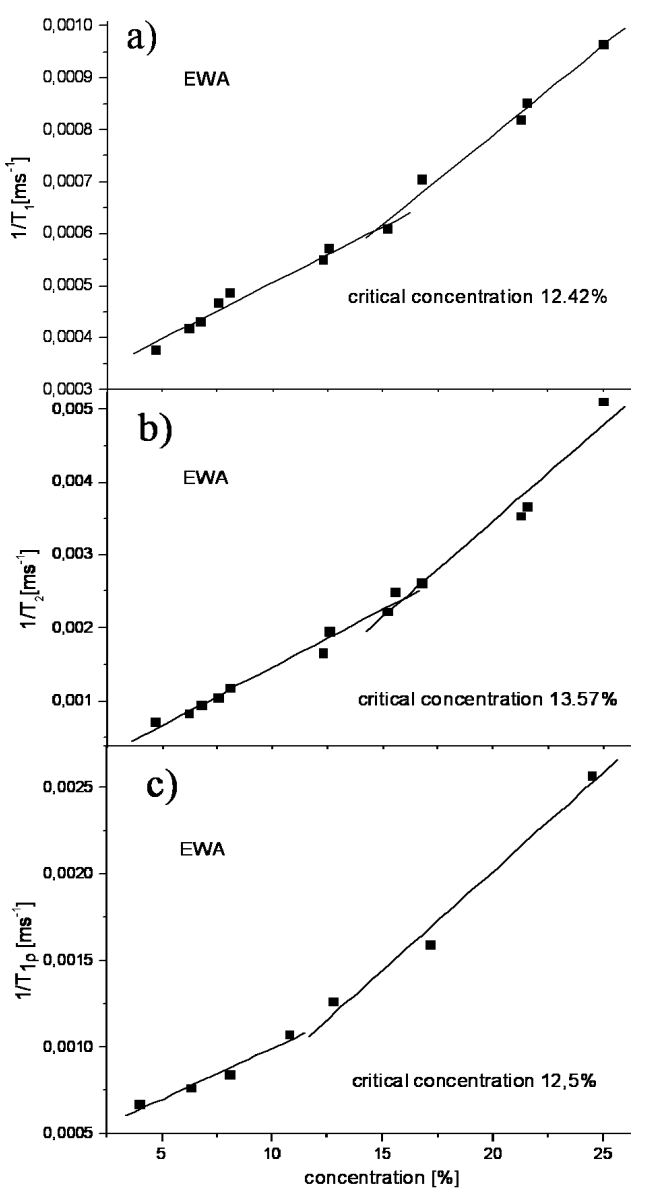

Fig. 2. (a) $R_{1}$, (b) $R_{2}$ and (c) $R_{1 \rho}$ dependences on concentration for EWA solutions.

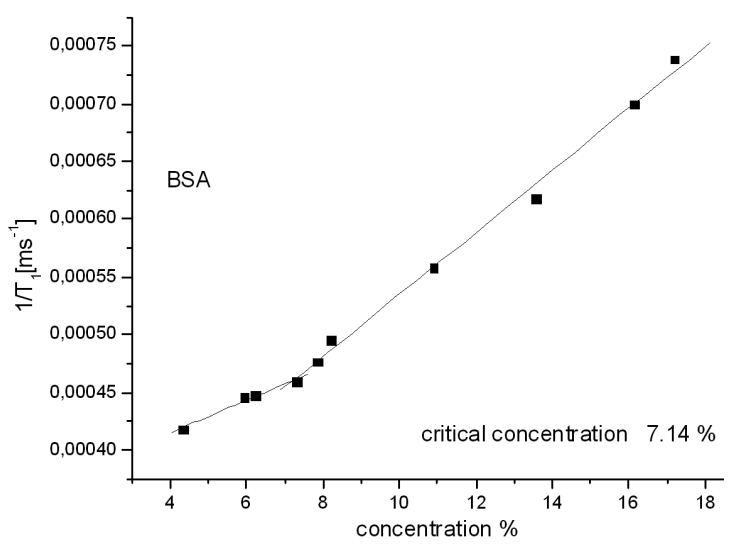

Fig. 3. $R_{1}$ dependence on concentration for BSA solutions.

lysozyme, EWA, and BSA were found by means of the concentration dependences plot analysis. These dependences can be well fitted linearly at low concentration $(<10 \%)$, according to known relation [6]:

$$
R_{i}=1 / T_{i \mathrm{w}}+k_{i} c,
$$

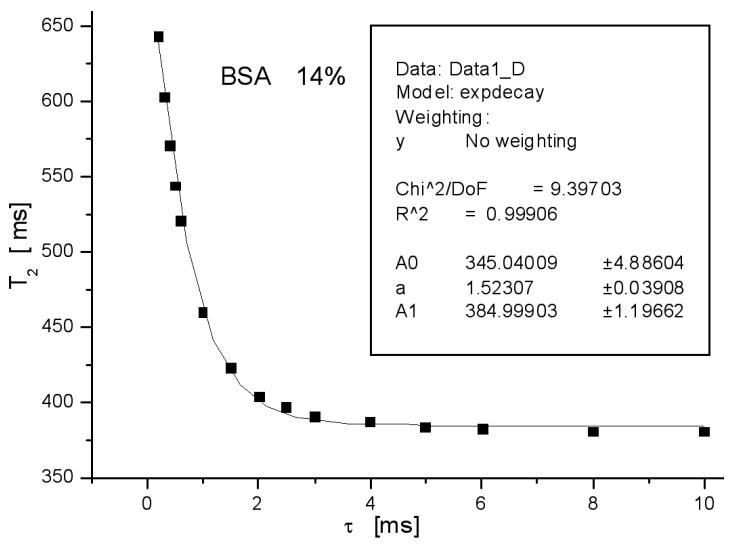

Fig. 4. CPMG $T_{2}$ dispersion for BSA solution $(14 \% \mathrm{w} / \mathrm{w})$ and one-exponential curve $T_{2}=A_{0}+$ $A_{1} \exp (-a / \tau)$ fitted to experimental data.

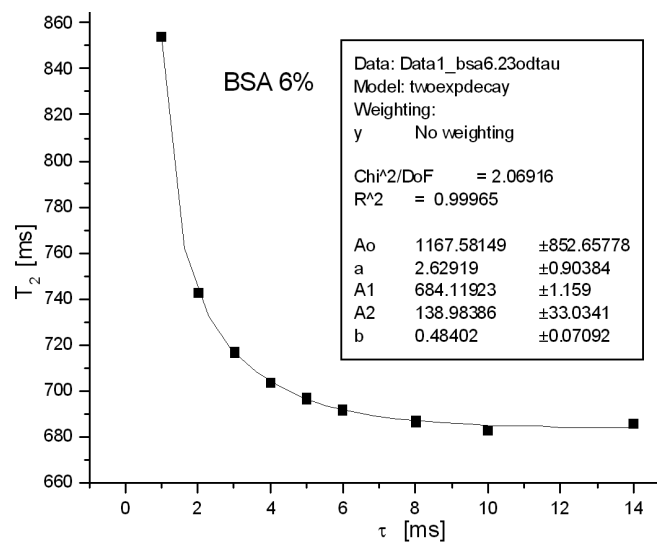

Fig. 5. CPMG $T_{2}$ dispersion for BSA solution $(6 \% \mathrm{w} / \mathrm{w})$ and two-exponential curve $T_{2}=A_{0}+$ $A_{1} \exp (-a / \tau)+A_{2} \exp (-b / \tau)$ fitted to experimental data.

where $T_{i \mathrm{w}}(i=1,2,1 \rho)$ are the relaxation times for pure water and $k_{i}$ are constants, which values are characteristic for each protein. At higher protein concentration the linear slopes bend and if a new linear fitting is plotted, the crossing of both lines determined the "critical" concentration: $(17 \pm 2) \%$ for lysozyme, $(12 \pm 2) \%$ for EWA and $(7 \pm 2) \%$ for BSA. The values of "critical" concentration depends on protein molecular weight, molecules' shape and also on intermolecular forces between water and protein molecules' surface and therefore are different [7]. If one assumes that protein molecule shape is almost spherical and density of, for example EWA and BSA, solutions are nearly the same at all concentrations, then the ratio of their "critical" concentrations $(1.73 \pm 0.6)$ should be equal to third root of the ratio of their molecular weights: $(66382 / 44287)^{1 / 3}=1.14$ [5]. Because of many factors not considered here, like asymmetry of protein molecules, and non-uniform hydration, protein aggregation and other experimental errors and etc., it seems that the agreement is satisfactory. 


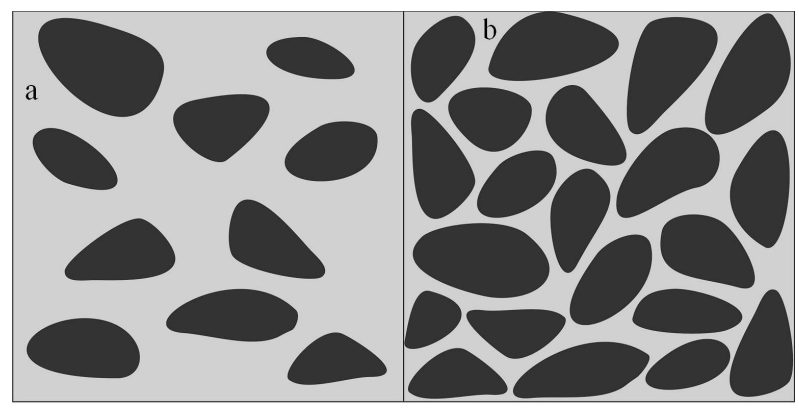

Fig. 6. Protein molecules in water solutions of concentrations lower (a) and higher (b) than "critical" one.

It is not accidental that values of "critical" concentration are very close to concentration, below which the two-exponential fit of CPMG dispersion function $T_{2}(\tau)$ starts (see Table). Exponential curves were fitted according to equations: $T_{2}=A_{0}+A_{1} \exp (-a / \tau)$ (one exponential) and $T_{2}=A_{0}+A_{1} \exp (-a / \tau)+A_{2} \exp (-b / \tau)$ (two exponential). As an example of $T_{2}$ dispersion the data for BSA are plotted and fitted in Figs. 4 and 5 for two solutions with concentrations higher and lower than "critical" one, namely $14 \%$ and $6 \%$.

Above "critical" concentration the protein molecules are close-packed and practically all water molecules are bounded to protein surface (Fig. 6) and move slower with correlation time of large protein molecule. The observed structure and molecular dynamics' changes affect the water-protein system and influences the relaxation processes. If the protein concentration increases, the amount of free water decreases and chemical exchange of water protons and the interaction between free and bounded water became difficult, only bounded water relaxation is observed in result. At lower concentration, when rate of free water increases, the two-component $T_{2}$ dispersion came to sight. Within limits of experimental errors the first parameter $a$ of two-exponential curves remain constant (for lysozyme $a=0.88 \pm 0.10$ or EWA $a=0.80 \pm 0.14$, and for BSA $a=0.65 \pm 0.12$ ) and its value is very close to value of the one-exponential curve parameter for pure water $(a=0.9 \pm 0.1)$. It means that this parameter can be related to the presence of free water fraction.

\section{Conclusion}

Performed investigations have shown the new opportunity of application of CPMG dispersion method for observation of protein hydration nature in solution. Subsequent measurements are being carried out and the results should be reported in the near future.

\section{References}

[1] D. Wierzuchowska, L.W. Skórski, B. Blicharska, Acta Phys. Pol. A 121, 434 (2012).

[2] R. Ishima, D.A. Torchia, J. Biomol. NMR 34, 209 (2006).

[3] I.R. Kleckner, M.P. Foster, Biochim. Biophys. Acta 1814, 942 (2011).

[4] D. Wierzuchowska, Ł. Żelazny, B. Blicharska, in: Ampere NMR School, Poznań/Wierzba (Poland), 2010, Book of Abstracts, p. 66.

[5] M. Singh, H. Chand, K.C. Gupta, Chem. Biodivers. 2, 809 (2005).

[6] O.K. Daszkiewicz, J.W. Hennel, B. Lubas, T.W. Szczepkowski, Nature 200, 1006 (1963).

[7] P. Kowalczyk, A. Ciach, P.A. Gauden, A.P. Terzyk, J. Colloid Interf. Sci. 363, 579 (2011). 